\title{
Innovative Methods To Improve Hospital Efficiency- Study Of Pneumatic Transport Systems (Pts) In Healthcare.
}

\author{
Dr. Arun. Mavaji. Seetharam ${ }^{1}$, Dr.Sudhakar.Kantipudi ${ }^{2}$,Dr.Somu.G ${ }^{3}$, Mr.Jibu $^{4}$ \\ ${ }^{I}$ (Department of Hospital Administration, Kasturba Medical college/ Manipal University, India) \\ 2(Office of Medical Superintendent, Kasturba Hospital, India) \\ 3(Department of Hospital Administration, Kasturba Medical college/ Manipal University, India) \\ 4(Office of Medical Superintendent, Kasturba Hospital, India)
}

\begin{abstract}
Increasing financial burden, onus on quality patient care and ever increasing competition has forced hospital administrators across world to focus on innovation in the Management of Hospitals. Logistics management is one such area. Internal logistics of materials like Laboratory samples, reports, blood products, drugs from pharmacy is of primary importance and has direct bearing on the provision of good quality care to patient and improving patient satisfaction. The present study was done to find the efficiency of one such method of logistics management, i.e. pneumatic transport system.

Methods: The study was conducted in a large tertiary care hospital for a period of 3 months. It includes study of available literature on pneumatic transport system .Data was collected by direct observation and comparing with existing conventional human based transportation.

Results: The study showed saving of a manpower and time of 94.6 Minutes for the total completion of process of sample transportation and Results being put online.

Conclusion: Pneumatic transport system is a valuable alternative to human based transportation. There is reduction in manpower usage, faster and safe transport of samples. However study noted there is cost escalation if installed in an existing structure and the system is more suitable for vertical structures.
\end{abstract}

Key Words: Innovation; Efficiency; Pneumatic Transport Systems; Healthcare

\section{Introduction:}

Introducing efficiency into an existing system is the key functions of all managers and its applicability to administrators in a healthcare scenario is no different. Current practice of evidence based management seeks to adopt known methods while inculcating innovative technologies as well.India is one of the few locations in the world with the latest in healthcare technologies including automation, surgical robotics, modular operating theatres, minimal access surgery systems, telemedicine and radiology.Although Indian hospitals and healthcare providers excel in the fields mentioned, intra-facility logistics and material-management remains untouched by latest innovations like automation of healthcare logistics, materials transportation and supply chain. Merely around US\$ one million is spent on intra-facility logistics (i.e. less than 0.06 per cent of the total spending). Although logistics comprises of almost 90 per cent of a healthcare facility's operations, it is considered least important in the 'list of procurements' and end up procuring out-dated, obsolete and redundant options. (1)

95 per cent of the total transport system with in the hospital are spontaneous in nature viz. transport of Drugs, Blood Samples, Organ \& Tissue Samples, Imaging Documents, Discharge Summary Documents, Billing Documents, Prescription Documents, Standard errands, Analysis Reports, Surgical materials and other samples. In-efficient and inadequate intra-facility logistics may increase costs on human resources, healthcare delivery and energy costs apart from other factors. This in turn causes several problems, increases risks and difficulties and consequently reduces the quality of patient care in the facility. (1)

Current methods used by the hospital for such Spontaneous transports are Human Based Transport (HBT) viz. using Ward Boys / Orderlies, Patient's Attendants / Relatives.In one of the studies conducted at a 600 bedded hospital facility it is estimated that over 300 such runs are made every day; of which 75 per cent are to and fro laboratories/blood bank; 10 per cent to and fro pharmacy / stores and 15 per cent to and from administration / billing.Dumb Waiters \& Elevators - High Energy Consumption and cause delays. (1)

With Budgetary constraints and Fledgling competitive situation hospitals are now facing new challenges: reduction of time patients spend in the hospital without loss in quality of care, reduction in staff costs and at the same time increasing results and efficiency. For these reasons the optimization of hospital logistics becomes more and more important. (8)

A study was conducted at a tertiary care teaching hospital with over 2000 beds in implementing the Pneumatic transport systems (PTS), its utilization and effectiveness over the conventional method of transport systems. 
1.1) Aim:To study the utilization effectiveness of Pneumatic Transport system in a tertiary Care Hospital

\section{2)Objectives:}

1. To study the implementation of pneumatic transport systems (PTS).

2. To study the utilization and effectiveness of PTS

3. Tostudy the benefits of PTS over human based transport (HBT)

\section{3)Pneumatic Transport System (PTS):}

Pneumatic tubes (or) capsule pipelines (or) Lamson tubes, also known as Pneumatic Tube Transport (PTT) are systems in which cylindrical containers are propelled through a network of tubes by compressed air or by partial vacuum. (1)

\section{4) Components of PTS}

\section{A. Blower:}

Large fans that move carriers through the tubes via vacuum and pressure. One or more three-phase blowers propel the carrier by means of compression or a vacuum created via a centrally controlled air switch. Self-adjusting Teflon gaskets in all system components guarantee airtight seals.

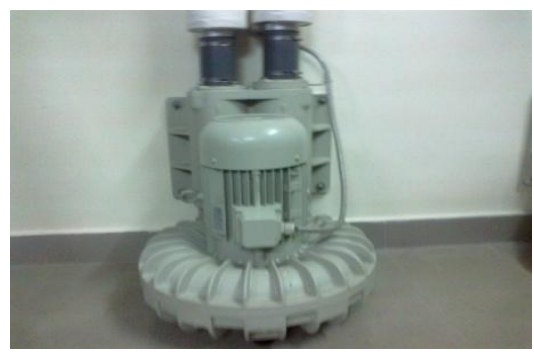

Figure 1: Blower

\section{B. Three-Way Diverter}

Three -way diverters are switching devices used at branching points within a tube network, allows carriers to travel between any two delivery stations. With a standardized offset, it transfers the carriers to the desired tube. The construction allows airtight, silent connection of the tubes.

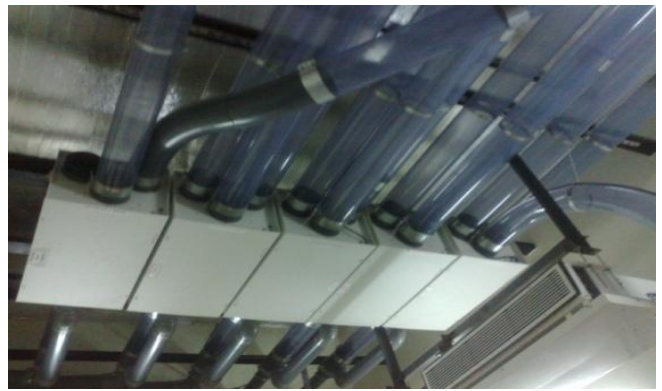

Figure 2: three-way diverter

\section{Carriers}

Carriers are reusable plastic containers that hold and protect items sent through a pneumatic tube system. Foam inserts can be placed inside a carrier for additional cushioning.

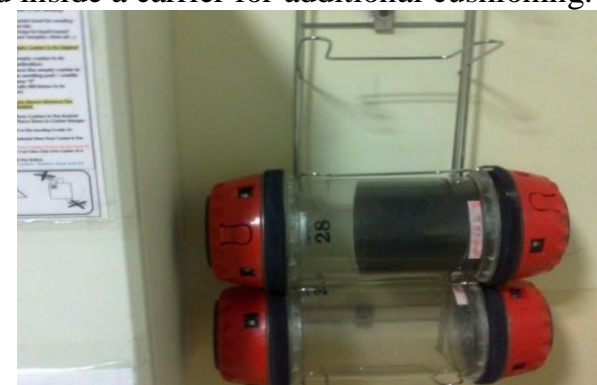

Figure 3: carrier 


\section{PVC tubes:}

PVC tubes are available in 4" (inches) and 6" (inches) diameter and mounted inside ceilings and mechanical rooms. They form a network through which carriers travel at speeds up to 17 miles per hour.

\section{E. Delivery stations:}

Delivery stations are positioned throughout the facility allowing personnel to send and receive carriers with the touch of a button.

\section{F. Computer control centre}

Computer control centre monitors all carrier traffic and calculates the fastest path for each transaction. To help manage traffic flow, systems are often divided into zones consisting of a few to a dozen stations each. Diverters and inter-zone tubes allow carriers to travel between zones. (3)

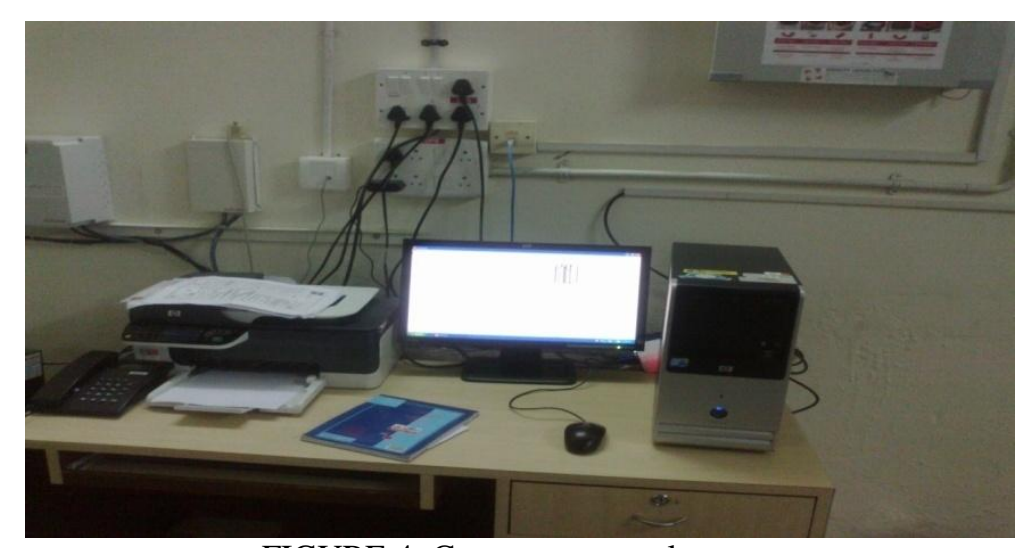

FIGURE 4: Computer control centre

\section{5)Advantages of PTS in Healthcare setting:}

- In a healthcare setting, a pneumatic tube delivery system allows for the fast, safe and reliable transport of pharmaceuticals, lab specimens, blood products and supplies throughout hospital clinical laboratories, pharmacies, emergency rooms, operating rooms, patient care areas and other locations much more quickly and safely than using a courier. Cost analyses by hospitals of all sizes show that pneumatic tube delivery systems cost less over time than employee couriers. (3)

- PTS can provide a safe efficient and rapid means of sending certain types of pathological specimens between hospital departments, from operation theatre and out-patients to the pathology laboratories. The use of these systems is increasing because they can improve specimen turnaround time so patients and hospital staff can receive test results quicker. They also allow for more effective time management of messenger staff by reducing the need to physically take specimens from one department to another. (4)

- The results of sending specimens through a computerized pneumatic air transport system and manually delivering specimens were compared for 15 chemical tests and six hematologic procedures. The specimens traversed a maximum of 829 feet (253 meters) involving 16 bends and eight transfer units at 25 feet/second (7.6 meters/second). Only the activity of lactate dehydrogenase exceeded the precision of the test in pneumatically transported specimens. (5)

- The pneumatic tube system decreased the median turnaround time for potassium and haemoglobin result on specimens from the emergency department by $25 \%$. The system evaluated is a rapid, efficient mechanism for sending specimens to the clinical laboratory that produces no significant effects on analytical results and has the ability to decrease turnaround time.(6)

- Using the principle of parallel processing for sample analysis, coupled with installation of a computerized pneumatic tube specimen delivery system, we provided improved turnaround time, a larger test menu, and 24-hour-a-day service from the central laboratory. As a result, the satellite laboratory in the Emergency Department was closed and 16 full-time equivalent positions were saved in the hospital. (7)

\section{6)Disadvantages and Risks involved in Human Based Transport (HBT):}

There are several risks and problems that entail Human Based Transport logistics:

- Delays: Hospital's staff carrying the materials may get diverted to a corner for a quick cigarette or choose to have a quick cup of tea with his or her friends - not realizing the critical life saving time being wasted; nonavailability of the staff at the time of need 
- During the physical carrying of tubesamples, the carrier staff may suddenly trip-over and the samples/materials fall off and break - which means the entire exercise needs to be repeated right from drawing the sample at the origin again, again loosing critical life saving time; mixing up of samples and materials is also common in such conventional mechanism, that may cause grave consequences and confusion in the course of procedure for the treatment of the patient.

- Theft: A universal problem, invariably theft is very common during transportation of drugs, instruments and other materials, etc.

- Exposure: There are many confidential and classified materials transported during a day in a hospital, which, are exposed to other unwanted personnel or people during human-transportation from one department to another.

- Bio-Hazard: Carrying of sample (blood/tissue etc...) personally involves high risk of exposure to the carrier of the materials of other persons in the facility to infections and subsequent cross-infections....many more risks and problems.

- $\quad$ Personnel: The Hospital needs to dedicate several staff members for just transporting and carrying samples and materials in the hospital - whereas they specifically hired for patient transportation, patient care etc... re-deputation of staff; over-hiring of staff, etc

- Energy: Use of dumb waiters and elevators for running such errands cause high consumption of energy; adding to already high energy bills of the facility; apart from causing delays due to long waits at the elevator doors.

However small these problems and risks may seem - they form a critical part of factors adversely affecting a hospital's operations and basic important goal- Good Patient Care!

In order to overcome the above problems and risks and to incorporate Automation \& Logistics Optimisation in Healthcare there is a unique solution available: Hospital Pneumatic Tube Systems - are economical, hi-tech, swift, secure and safe solution. (3)

\section{7)Pneumatic transport system for an already established set up:}

Pneumatic tube systems don't necessarily have to be installed at the outset of construction. Even in an already operating hospital, a pneumatic tube system can be easily integrated. Depending on the various structural conditions, the system can be laid inside or outside the walls. Other factors, for instance system capacity, load size or weight, are taken into consideration as well. (3)

\section{8)Specific demands of a hospital:}

Since a hospital is not a typical working place, its pneumatic tube system has to fulfil a series of high demands:

- In order to guarantee a safe transport of laboratory samples, the carrier speed may be modified. Low-speed transport is selected manually or automatically by choosing a certain dispatch or receiving station.

- The carriers can be adapted to hold different types of insert pockets or test tube holders. Arriving carriers are braked carefully upon arrival and sent directly into the tube without a jolt. The system functions silently and is patient-friendly.

- Transport loads with restricted access can be secured by means of a code

- Thermo chemical sterilization of the carriers to be carried out manually or automatically.

- The system is hermetically sealed.

- The computerized control system permits statistical evaluations of the traffic data, e.g. the number of transports, for itemized cost analysis and billing. (3)

\section{9)Installation of Pneumatic transport system at Kasturba Hospital:}

Kasturba Hospital, Manipal is a tertiary Care Hospital with 36 disciplines providing Quality Health care under the Kasturba Medical College Manipal. It is a 2050 Bedded Hospital with an aim to provide faster and quality Care, planned the installation of Pneumatic Transport System with 2 Critical Blocks of the Hospital covering 650 Beds including Central medical ICU, Multidisciplinary ICU and Orthopaedics Triage and Post/Pre Op ICUs

\section{Initial Planning:}

\section{Steps in implementation:}

Study on the existing System of Transportation and time taken for the Transports was started from Dec 2009. A 3-month period Sample Transportation; Manpower Utilization and Time Taken were studied to put up the Proposal for Installation of the PTS in the hospital 


\section{The study showed the following Observations:}

Table-1

\begin{tabular}{|l|l|l|l|l|}
\hline $\begin{array}{l}\text { Number of samples } \\
\text { transported per day }\end{array}$ & From & To & Distance & Time taken \\
\hline 1500 & $\begin{array}{l}\text { Women and child } \\
\text { block }\end{array}$ & Clinical laboratory & $2 \mathrm{kms}$ & 1.5 hours* \\
\hline 650 & Main hospital & $\begin{array}{l}\text { Biochemistry } \\
\text { laboratory }\end{array}$ & $3 \mathrm{kms}$ & 2.5 hours* \\
\hline
\end{tabular}

*(Average of Total Timings and received logs from Labs)

\section{Manual transportation Problems:}

- Additional Requirement of 15 Manpower to transport samples.

- Errors of Sample Mismatch with slips when received by concerned Lab

- Reporting Time of 137 min after sample drawn from a patients affecting Clinician and clinical diagnosis

- Patient dissatisfaction including Nursing staff dissatisfaction as the Nurses needs to search the personals for the transportation

Table-2: Typical Scenario of time taken by HBT Vs PTS

\begin{tabular}{|c|c|c|c|c|c|c|}
\hline $\begin{array}{l}\text { Drawing } \\
\text { of } \\
\text { Sample } \\
\text { from } \\
\text { Patients }\end{array}$ & $\begin{array}{l}\text { Slips and } \\
\text { arrangement } \\
\text { by Lab Staff } \\
\text { to send to } \\
\text { Clinical Lab }\end{array}$ & $\begin{array}{l}\text { Packing and } \\
\text { arranging } \\
\text { manpower }\end{array}$ & $\begin{array}{l}\text { Handing over } \\
\text { samples to } \\
\text { the GD } \\
\text { worker with } \\
\text { dispatch slip }\end{array}$ & $\begin{array}{l}\text { Transportation } \\
\text { to Clinical Lab } \\
\text { and taking } \\
\text { receipt slip }\end{array}$ & $\begin{array}{l}\text { Reporting } \\
\text { of Results } \\
\text { after } \\
\text { tests }\end{array}$ & $\begin{array}{l}\text { Total Hrs. for } \\
\text { the patient to } \\
\text { get after } \\
\text { sample } \\
\text { collection }\end{array}$ \\
\hline $2.0 \mathrm{Min}$ & 5.0 Min. & 25.0Min & $10.0 \mathrm{Min}$ & $60.0 \mathrm{Min}$ & 35.0Min & $137.0 \mathrm{Min}$ \\
\hline & & & \multicolumn{2}{|c|}{$\begin{array}{l}\text { PTS Transporting Time Taken } \\
\text { after Installation of PTS in June } \\
13^{\text {th }} 2011\end{array}$} & & \\
\hline $2.0 \mathrm{Min}$ & $5.0 \mathrm{Min}$ & Nil & \multicolumn{2}{|c|}{.66 Min } & $35.0 \mathrm{Min}$ & 42.6 Min \\
\hline & & & \multicolumn{2}{|c|}{ Difference/Savings of Time } & & 94.6 Min \\
\hline
\end{tabular}

The above table shows a saving of a manpower and time of 94.6 Minutes for the total completion of process of sample transportation and Results being put online.

\section{Process for vendor selection:}

Tenders were called for installation of Pneumatic transport system in the Hospital. Vendor was selected on the basis of Technical superiority and speedy completion of the project. A team from the hospital visited different hospitals were the system was installed by the chosen vendor to assess the functionality and feasibility of the system.

\section{Pre implementation difficulties:}

The hospital has a vast horizontal expanse with inter connected blocks at various levels. Such a kind of design poses problems for routing of pipes; distance between biochemistry laboratory and the hospital posed operational problems; Due to heavy patient and public traffic flow during daytime, most of the work had to be done at night time which invariably posed threat to the execution time of the project; Installation had to be done in existing structure; Care had to be taken to shift electrical and plumbing fixtures; In addition there was a cost escalation of $10-20 \%$ on account of repairs and maintenance.

\section{End user problems:}

Training of staff took nearly 20-30 days; the break-in period for staff to get accustomed to the new system was another 30 days; wrong dialling of stations was also routinely encountered, if the stations number is not dialled properly, chances that the carrier gets stuck at the junction impeding the flow of other carriers.

PROJECT COST

\begin{tabular}{|l|l|}
\hline Equipment cost & Rs 76,00,000 \\
\hline Projects Civil/Electrical/Control Room Creation & Rs 20,00,000 \\
\hline Total Project Cost & Rs 96,00,000 \\
\hline
\end{tabular}




\section{Conclusion:}

Pneumatic transport system (PTS) is a valuable alternative to conventional human dependant transport. The study shows there is definite saving in manpower and also the time for transport by nearly 96 minutes. With increasing emphasis on effective utilization of resources available in order to cut operational cost in hospitals, it is ideal for modern hospitals and healthcare settings to switch over to using these systems. The study also brings to light the cost escalation incurred in installing PTS into existing buildings. Hence it is better to consider for these facilities at the hospital planning stages itself. Finally although PTS systems can be a boon to any type of hospital buildings it is ideal for vertical structures.

\section{References:}

[1]. http://www.expresshealthcare.in/200901/valueadd02.shtml (accessed on 10-6-2012)

[2]. "Gone with the wind: Tubes are whisking samples across hospital". Stanford School of Medicine.2010-01-11 (accessed on 10-6-2012)

[3]. www.swisslog.com/index/hcs-index/hcs-systems/hcs-pts.htm (accessed on 10-6-2012)

[4]. http://www.hse.gov.uk/pubns/misc186.pdf. (Accessed 4th June 2012)

[5]. Weaver DK, Miller D, Leventhal EA, Tropeano V, Evaluation of a computer-directed pneumatic-tube system for pneumatic transport of blood specimens, American Journal of Clinical Pathology. 1978 Sep; 70(3):400-5.

[6]. Keshgegian AA, Bull GE, Evaluation of a soft-handling computerized pneumatic tube specimen delivery system. Effects on analytical results and turnaround time, American Journal of Clinical Pathology. 1992 Apr; 97(4):535-40

[7]. Green M, Successful alternatives to alternate site testing. Use of a pneumatic tube system to the central laboratory, Arch Pathol Lab Med 1995 Oct; 119(10):943-7

[8]. Pneumatic Tube System Logistics in Hospitals, www.swisslog.com (accessed on 7th July, 2012) 